\title{
TANDEM-X MISSION: OVERVIEW AND EVALUATION OF INTERMEDIATE RESULTS
}

\author{
U. Soergel, K. Jacobsen, L. Schack
}

\author{
Institute of Photogrammetry and GeoInformation, Leibniz Universität Hannover, Germany - \\ \{soergel | jacobsen | schack\}@ipi.uni-hannover.de
}

WG VII/2

KEY WORDS: SAR, DEM/DTM, Interferometer, Environment, Accuracy

\begin{abstract}
:
The German Aerospace Center (DLR, Deutsches Zentrum für Luft- und Raumfahrt) currently conducts the bistatic interferometric synthetic aperture radar (SAR) Mission TanDEM-X, which shall result in a DEM of global coverage in an unprecedented resolution and accuracy according to DTED level 3 standard. The mission is based on the two SAR satellites TerraSAR-X and TanDEM-X that have been launched in June 2007 and 2010, respectively. After the commissioning phase of TanDEM satellite and the orbital adjustment the bistatic image acquisition in close formation began end of 2010. The data collection for the mission is scheduled to last about three years, i.e., the bigger part of the required data have been already gathered. Based on this data DLR will conduct several processing steps in order to come up finally with a global and seamless DEM of the Earth's landmass which shall meet the envisaged specifications. Since the entire mission is an endeavor in the framework of a private-public-partnership, the private partner, Astrium, will eventually commercialize the DEM product. In this paper, we will provide an overview of the data collection and the deliverables that will come along with TanDEM-X mission. Furthermore, we will analyze a DEM derived from early stage immediate products of the mission.
\end{abstract}

\section{INTRODUCTION}

DEM generation based on interferometric SAR (InSAR) requires at least two complex SAR images which feature an across-track baseline of suitable length. The topographic height information is derived from the phase difference (i.e., the interferogram) of the coregistered individual SAR images (Soergel, 2010).

Space borne SAR data are usually acquired in so-called repeatpass mode, this means that the imagery are taken from the same orbit but separately at different dates, the time lapse is usually an integer multiple of the revisit cycle of the given satellite in use. Such sequential data acquisition comes along with two major drawbacks: Firstly, in the period between data takes the scene can change and such temporal decorrelation even may result in entire loss of coherence. Besides real changes of land cover already subtle processes like growth of plants during phenological active periods can significantly deteriorate DEM quality leading to areas of pure noise in extreme case.

Secondly, water vapor in the troposphere influences the velocity of light. This effect is characterized by a spatial correlation length in the order of kilometers, whereas it fully decorrelates in the repeat cycle time span of common low Earth orbit remote sensing satellites. Therefore, the atmospheric path delay of the signal caused by this effect will differ for image acquisitions taking place at dates separated by some weeks, a nuisance term superimposes the topographic phase term of interest.

Both drawbacks are avoided if the two SAR images are acquired simultaneously: no temporal changes can occur and whatever the atmospheric conditions are, the related phase term cancels out because of taking the image difference. Such singlepass data are commonly gathered using airplanes which are equipped with two antenna systems mounted in a manner that an across-track baseline is established.

One famous exception was SRTM, an US, German, and Italian Space Shuttle mission lasting for 11 days in the year 2000
(Rabus et al., 2003). The baseline was realized using a fixed boom of $60 \mathrm{~m}$ length. From the collected SAR data a DEM of about $30 \mathrm{~m}$ grid size was derived which covers the global land mass in the latitudes from $56^{\circ} \mathrm{S}$ to $60^{\circ} \mathrm{N}$, but in the WEB only a DEM reduced to approximately $90 \mathrm{~m}$ point spacing is available.

Since end of 2010 DLR conducts the InSAR mission TanDEM$X$. In contrast to SRTM not a single sensor carrier is in use but a constellation of two individual satellites called TerraSAR-X and TanDEM-X launched in summer 2007 and 2010, respectively. After the commissioning phase and the orbital adjustment of the TanDEM-X satellite the bistatic image acquisition in close formation began end of 2010. In order to enable save spacecraft navigation, the orbits of the satellites must never cross. Hence, the orbits are chosen such that the satellites follow a helix configuration (see Figure 1). As a consequence, depending on the latitude the spatial baseline changes constantly which has to be measured with millimeter accuracy to meet the specifications of the DEM product. Vanishing effective baselines in acrosstrack direction for some latitudes would prevent DEM generation there. To account for this, the helix set-up was chosen such that either for ascending or descending orbits a sufficiently large effective baseline is ensured. The baseline configuration was adjusted in the second year leading to larger baselines by a factor of about 1.3 . One reason was to raise the sensitivity at the cost of smaller unambiguous height span (i.e., the $2 \pi$ of the wrapped differential phase scale to a smaller elevation span). However, the idea is to incrementally improve the coarser DEM of the first year that in turn features a larger unambiguous height. In such manner phase-unwrapping is supported for the second year data also.

The helix configuration leads to a significant along-track baseline, too. Its magnitude is between $0 \mathrm{~m}$ in polar regions up to $1000 \mathrm{~m}$ at the equator (Wendleder et. al, 2013). Such set-up is useful to determine the velocity of objects like cars, but with respect to the DEM generation it is disadvantageous because of 

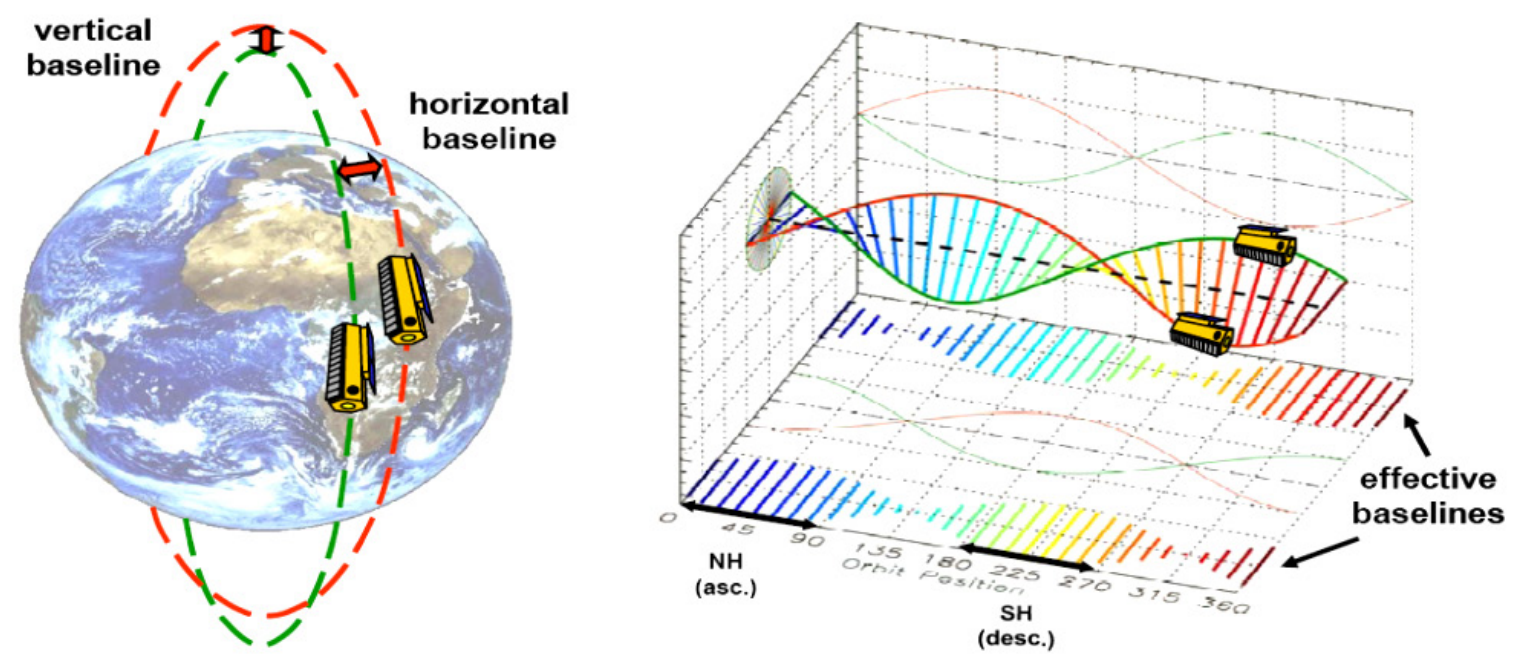

Fig. 1: The Helix configuration of the two satellites leads to varying horizontal and vertical baselines. Therefore, the effective baseline for DEM extractions is a function of latitude (TanDEM Science Plan, courtesy of DLR).

the time delay of up to 50 milliseconds. The problem is that water surfaces usually already fully decorrelate after just a few milliseconds. This is only not the case at very high latitudes where the water is frozen anyway. As a consequence, no useful signal is received from surfaces of liquid water like the ocean, lakes, or rivers - at the related locations there is noise only. In order to mask out such areas much effort is spend from DLR to detect them in an automated manner (Wendleder et. al, 2013). This problem did not occur as pronounced for SRTM because the fixed along track baseline of $7 \mathrm{~m}$ lead to 0.5 milliseconds time lag only.

The data acquisition for the mission is scheduled to last about three years, which means the bigger parts of the required data have been already collected. In parallel and afterwards the DLR conducts several processing steps in order to come up finally with a global and seamless DEM of the Earth's landmass. According to the TanDEM-X Science Plan (2010) the mission is divided in four phases:

1. In the first year SAR data for a global DEM were acquired with small baselines.

2. In the second year a second global coverage with larger baseline took place. In the standard case from those two DEM the final product shall be derived.

3. During the first half of the third year DEM data takes for difficult terrain with different viewing geometry are scheduled.

4. In the additional mission lifetime customized DEM with large baselines are planned.

The first two phases have already been accomplished. In this paper, we will discuss various data acquisition aspects, TanDEM products and deliverables. Since the entire mission is an endeavor in the framework of a private-public-partnership, the private partner, Astrium, will eventually commercialize the DEM product. Of course, this product will be available only after the end of the data acquisition and some processing. However, during the mission DLR provides the radar community intermediate data for scientific purpose. In the last part of this paper, we compare a DEM derived from a single bistatic TanDEM-X dataset for the test scene Hannover.

\section{OVERVIEW OF THE TANDEM-X MISSION}

\subsection{DEM products and auxiliary data}

In Table 1 are various intermediate and DEM products summarized (TanDEM-X Science Plan, 2010). The accuracy numbers refer to the $90 \%$ quantile of linear error (LE90). Besides the standard product, abbreviated as TDX DEM, also an intermediate global DEM (IDEM) shall be provided, which is based exclusively on the data acquired during the first year. TSX DEM and IDEM are expected to be available 4 years and 2 years after launch of TanDEM-X, respectively. Both products shall be offered in additional versions with reduced spacing of factors 2 and 4 but in turn lower relative error. On the other hand, in some cases even a finer grid is desired. To meet such demand, so-called FDEM and HDEM are foreseen, which are however scheduled to be processed and delivered later.

Please note, that the digital elevation models (DEM) represent rather the surface of the scene (e.g., buildings) than the underlying terrain. Due to the small signal wavelength of only 3 $\mathrm{cm}$ this is true for vegetation, too. Hence, we rather deal with digital surface models (DSM) than with digital terrain models (DTM).

In the last row of the table the CoSCC data are presented. This is not any DEM but an intermediate product: it consists of two coregistered complex SAR images which were acquired simultaneously by the two satellites. In contrast to all other products, this kind of data is already available for the scientific community. The results for our test scene discussed later are based on such data.

The height value of the final DEM will be a weighted average of all independent height values (at least two). In addition to the DEM products the DLR will provide a number of useful auxiliary data (TanDEM-X DEM Product Specification, 2013):

- Height error map (HEM): For each DEM pixel the corresponding height error is given in form of the standard deviation, which relies on the interferometric coherence and geometrical considerations.

- Water indication mask (WAM): Based on two amplitude and one coherence threshold such areas are detected automatically (Wendleder et. al, 2013). The aim is to preserve islands that are larger than 1 hectare and to indicate as many water bodies of size larger 


\begin{tabular}{|l|c|c|l|c|}
\hline Product & Spatial resolution & $\begin{array}{c}\text { Absolute Vertical } \\
\text { Accuracy (LE90) }\end{array}$ & $\begin{array}{l}\text { Relative Vertical } \\
\text { Accuracy (LE90) }\end{array}$ & Availability \\
\hline TDX DEM & $12 \mathrm{~m}$ & $<10 \mathrm{~m}$ & $\begin{array}{l}2 \mathrm{~m} \text { (slope }<20 \%) \\
4 \mathrm{~m} \text { else }\end{array}$ & 4 years after launch \\
\hline IDEM & $12 \mathrm{~m}$ & $\sim 10 \mathrm{~m}$ & $\begin{array}{l}2 \mathrm{~m}(\text { slope }<20 \%) \\
4 \mathrm{~m} \text { else }\end{array}$ & $\begin{array}{c}2.5 \text { years after } \\
\text { launch }\end{array}$ \\
\hline FDEM & $6 \mathrm{~m}$ & $<10 \mathrm{~m}$ & $\begin{array}{l}4 \mathrm{~m}(\text { slope }<20 \%) \\
8 \mathrm{~m} \text { else }\end{array}$ & $\begin{array}{c}\text { Later, on request } \\
\text { only }\end{array}$ \\
\hline HDEM & $6 \mathrm{~m}$ & $<10 \mathrm{~m}$ & Up to $0.8 \mathrm{~m}$ & $\begin{array}{c}\text { Later, on request } \\
\text { only }\end{array}$ \\
\hline CoSSC & StripMap Mode & - & - & Available \\
\hline
\end{tabular}

Tab. 1: Products of TanDEM-X Mission (TDX). Both the TDX DEM and the IDEM (intermediate DEM) shall be in addition provided with reduced resolution by factor 2 and 4 which in turn feature improved relative accuracy. In contrast FDEM (finer pixel spacing) will lead to larger lower relative accuracy, whereas HDEM is a high resolution DEM for which additional image pairs have to be taken with larger baselines. FDEM and HDEM will be delivered after the standard product. Finally, the CoSSC is not a DEM but a corigistered complex image pair in slant range geometry.

than 2 hectares as possible. The water areas are detected in each interferogram and those results are combined to the water indication mask.

- $\quad$ Coverage map (COV): The InSAR measurements are taken with overlap in range direction and repeatedly over time. Those data are formed to a mosaic and tailored to tiles of $1^{\circ} \times 1^{\circ}$ extension. The coverage map indicates how many height values from different DEM acquisitions were used.

- Amplitude mosaic (AMP SAR, mean value): Amplitude mosaics are useful to support the thematic interpretation of the data. This product provides the mean amplitude.

- Amplitude mosaic (AM2 SAR, minimum value): Sometimes it is desired rather to look at the minimum amplitude, for example, if in one acquisition a disturbing very strong reflection occurred.

Further add-on layers which may accompany the final product according to TanDEM-X DEM Product Specification (2013) are a so-called consistency mask that would indicate regions with at least one deviating interferogram, the layover \& shadow mask that is very useful in case of undulated terrain, and finally an interpolation mask.

\subsection{Error budget}

After some pre-processing in terms of up-sampling of the complex SAR images $u$ to avoid aliasing in the subsequent interferogram generation, spectral filtering to the common signal band, and coregistration the interferogram is calculated:

$$
\begin{aligned}
& s=u_{1} \cdot u_{2}^{*}=\left|u_{1}\right| \cdot e^{j \varphi_{1}} \cdot\left|u_{2}\right| \cdot e^{-j \varphi_{2}}=\left|u_{1}\right| \cdot\left|u_{2}\right| \cdot e^{j \Delta \varphi} \\
& \text { with } \Delta \varphi=W\left\{\varphi_{f E}+\varphi_{\text {Topo }}+\varphi_{\text {Defo }}+\varphi_{\text {Error }}\right\} \approx \frac{2 \pi \cdot p}{\lambda} \cdot \Delta r
\end{aligned}
$$

We consider here only the term dealing with the topography. There is an approximate linear function between the difference phase $\Delta \varphi$ and the offset $\Delta h$ of an object from a reference altitude:

$$
\Delta h \approx \frac{\lambda}{2 \pi \cdot p} \cdot \frac{r \cdot \sin (\theta)}{B_{\perp}} \cdot \Delta \varphi, B_{\perp}=B \cdot \cos (\theta-\xi),
$$

with $p=1$ or 2 depending on the InSAR mode, range $r$, and wavelength $\lambda$. Replacing $\Delta \varphi$ with $2 \pi$ gives the so-called ambiguous height $h_{a m b}$, this means, in case the height variation of the topography exceeding this value, we have to conduct phase-unwrapping to reconstruct the gradient properly.

To some degree the local DEM accuracy can be assessed a priory from the coherence of the given SAR data. The term coherence is defined as the complex cross-correlation coefficient of the SAR images, for many applications only its magnitude (range [0...1]) is of interest. Coherence is usually estimated from the data by spatial averaging over a suitable area covering N pixels:

$$
\gamma=\frac{E\left[u_{1} \cdot u_{2}^{*}\right]}{\sqrt{E\left[\left|u_{1}\right|^{2}\right] \cdot E\left[\left|u_{2}\right|^{2}\right]}}=|\gamma| \cdot e^{j \Phi_{0}},|\gamma| \approx \frac{\left|\sum_{n=1}^{N} u_{1}^{(n)} \cdot u_{2}^{(n) *}\right|}{\sqrt{\sum_{n=1}^{N}\left|u_{1}^{(n)}\right|^{2} \cdot \sum_{n=1}^{N}\left|u_{2}^{(n)}\right|^{2}}}
$$

Low coherence magnitude values indicate poor quality of the height derived by InSAR, whereas values close to one coincide with accurate DEM data. Several factors may cause loss of coherence (Hanssen, 2001): non-overlapping spectral components in range $\left(\gamma_{\text {geom }}\right)$ and azimuth (Doppler Centroid decorrelation, $\left.\gamma_{D C}\right)$, volume decorrelation $\left(\gamma_{v o l}\right)$, thermal noise $\left(\gamma_{\text {thermal }}\right)$, temporal decorrelation $\left(\gamma_{\text {temporal }}\right)$, and imperfect image processing $\left(\gamma_{\text {processing, }}\right.$, e.g., coregistration and interpolation errors). Usually those factors are modeled to influence the overall coherence in a multiplicative way:

$$
\gamma=\gamma_{\text {geom }} \cdot \gamma_{D C} \cdot \gamma_{\text {vol }} \cdot \gamma_{\text {thermal }} \cdot \gamma_{\text {temporal }} \cdot \gamma_{\text {proces } \sin g}
$$

In case of single-pass Interferometry neither atmospheric delay nor scene decorrelation have to be taken into account, because both images are acquired at the same time. The quality of such DEM is mostly governed by the impact of thermal noise, which is modeled to be additive, i.e., the two images $u_{i}$ consist of a common deterministic part $c$ plus a random noise component $n_{i}$. Then, the coherence is modeled to approximately be a function of the local signal to noise ratio (SNR):

$$
|\gamma| \approx \frac{1}{1+\frac{1}{S N R}} \text {, with SNR }=\frac{|c|^{2}}{|n|^{2}}
$$



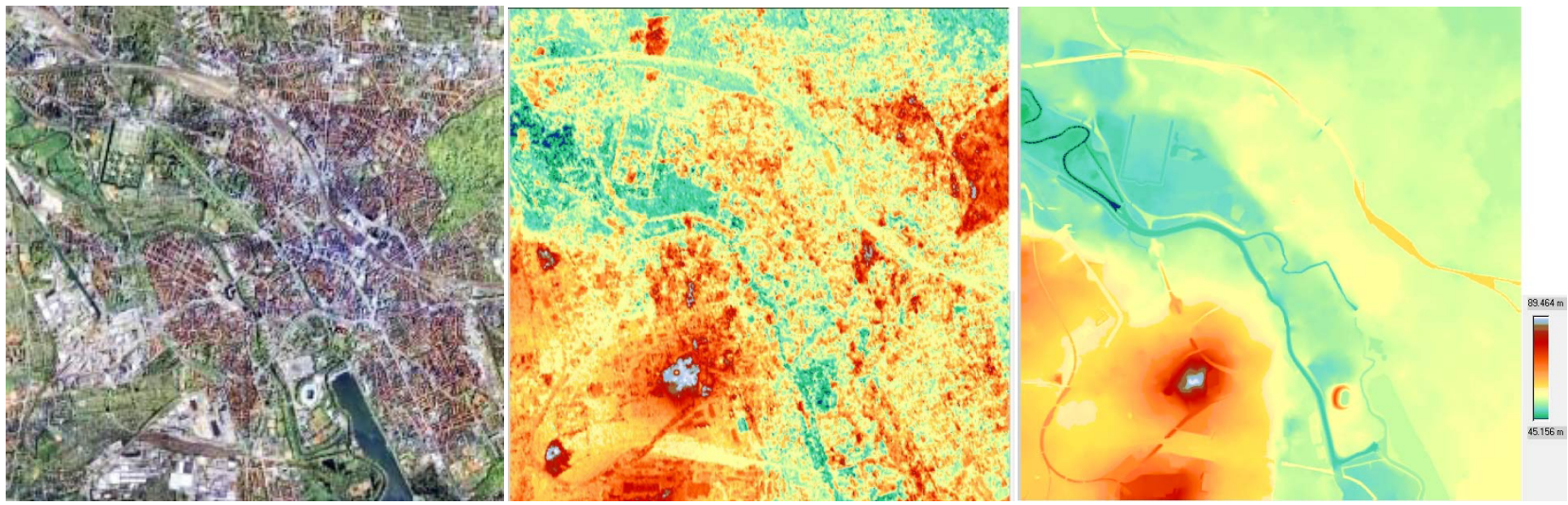

Fig. 2: Subset Inner city area of Hannover: Google image, CoSCC DSM, Reference DTM

Martone et al. (2012) report for most land cover classes an SNR better than $6 \mathrm{~dB}$ and typical values of $\gamma$ for the TanDEM-X missions are in the range of 0.6-0.8. However, this means in order to meet the required height accuracies given above the data have to be averaged or equivalently so-called multi-looking has to take place (Krieger et al., 2007) because the standard deviation $\sigma_{\varphi}$ of the phase approximately depends both on the SNR and the number of looks $L$ :

$$
\sigma_{\varphi} \approx \frac{1}{\sqrt{S N R} \cdot \sqrt{L}}
$$

In summary, this is the reason why the grid size of the DEM was chosen to $12 \mathrm{~m}$, which coincides with the required number of looks to meet the specifications. Depending on topography between 15 and 25 samples of the original slant range SAR data are used for this purpose (TanDEM-X DEM Product Specification, 2013).

\section{ANALYSIS OF AN INTERMEDIATE PRODUCT}

We are using a CoSSC image pair that was acquired $14^{\text {th }}$ of May 2012 in alternating bistatic mode over the region Hannover. The baseline configuration is such that $h_{a m b}$ is $16 \mathrm{~m}$ only, therefore despite flat terrain we expect phase unwrapping issues in particular at high buildings. Besides the raw data and meta information in form of xml-files DLR provides useful socalled quicklooks, which are given as significantly downsampled images.

The estimated coherence is in the range [0.24 -0.99$]$ with $\mu=$ 0.73 and $\sigma=0.13$, those numbers confirm the values reported by Martone et al. (2012). Looking at the coherence image there are also some agricultural fields with even higher coherence, whereas we observe lower values in vegetated areas and for water surfaces like "Maschsee" in the middle of the scene (appears also dark in amplitude image). We will discuss some of these issues in more detail later.

We have processed the data with the commercial software SARscape in ENVI environment. Since signal processing is not the scope of this paper, we just used the standard method to derive a geocoded DSM from the given SAR data. Eventually, we produced a geocoded DSM of $8 \mathrm{~m}$ spacing. In order to avoid confusion, we will call this product CoSCC DSM. Please note, we deal here with an intermediate product and NOT any official product described in Section 2. Therefore, the quality numbers are expected to be better for the real final products. For the test we focus on the city area of Hannover in the center of the scene and we use a DTM and a LIDAR DSM for comparison.

\subsection{Validation versus DTM}

We use a DTM from survey administration as reference $(6 \mathrm{~km} \mathrm{x}$ $6 \mathrm{~km}$, spacing $12.5 \mathrm{~m}$, and SZ 0.25-0.5 m). In Figure 2 the elevation models and a Google image for better interpretation are shown. It is clear at first glance that we deal here with a DSM and a DTM. The discrepancies nicely coincide with large vegetated areas in particular in the East (urban forest "Eilenriede") and building locations.

We are applying our own software BLUH (Jacobsen, 2008) which is also able to account for systematic shifts. In the frame work of the investigation some accuracy figures are derived to assess the quality of the CoSSC DSM:

- $\quad$ RMSZ = root mean square difference of analyzed DEM against reference DEM

- Bias = systematic height difference (linear mean of differences)

- $\mathrm{SZ}=$ standard deviation of Z (RMSZ improved by bias)

- $\mathrm{MAD}=$ Median absolute deviation - Median of absolute values of Z-discrepancies

- $\quad$ NMAD $=$ normalized median absolute deviation $=$ MAD * 1.48 - MAD has $50 \%$ probability level, multiplied with 1.48 corresponds to $68 \%$ probability level - in case of normal distributed discrepancies are NMAD and SZ identical. NMAD is based on linear values while $\mathrm{SZ}$ is based on square sum, so in case of a higher number of larger discrepancies, SZ will be larger as NMAD (Höhle and Höhle, 2009).

- $\quad$ LE90 = linear error $90 \%$, by definition a threshold value of $90 \%$ of the absolute values of the discrepancies. In case of normal distributed discrepancies LE90 $=\mathrm{SZ} * 1.65$

- $\quad$ LE95 = as LE90, but with a threshold of $95 \%$

The accuracy does not show any dependency upon aspects, this mainly is caused by the fact that the test area is dominantly flat. This is also the reason why no slope dependency is observed. The following two tables provide information about the accuracy figures on the next page and a detail analysis of the left and right branches of the unsymmetrical distribution. The latter is shown in Figure 4 next to a color coded difference image (Figure 3).

\begin{tabular}{|c|c|c|c|c|c|}
\hline RMSZ & bias & SZ & NMAD & LE90 & LE95 \\
\hline $3.81 \mathrm{~m}$ & $-0.25 \mathrm{~m}$ & $3.80 \mathrm{~m}$ & $2.87 \mathrm{~m}$ & $5.71 \mathrm{~m}$ & $8.42 \mathrm{~m}$ \\
\hline
\end{tabular}




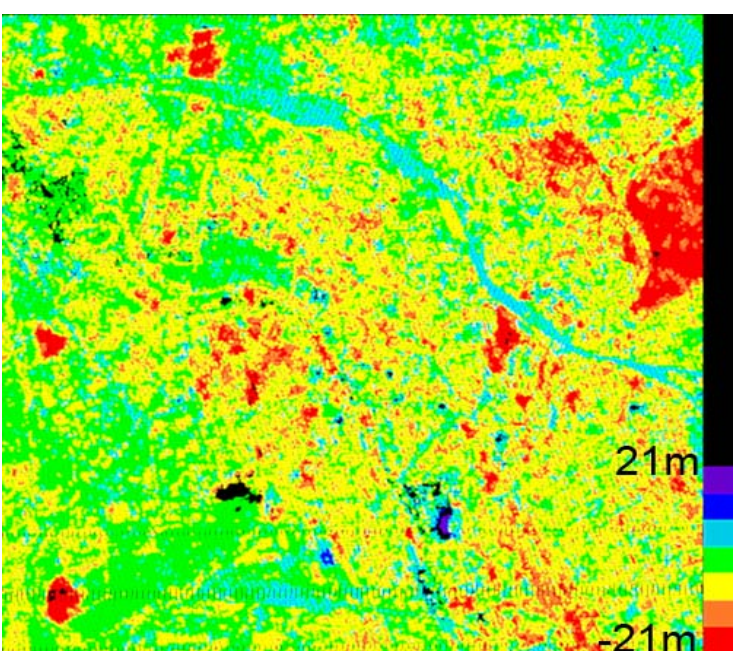

Fig. 3: Color coded difference reference DTM CoSSC DSM

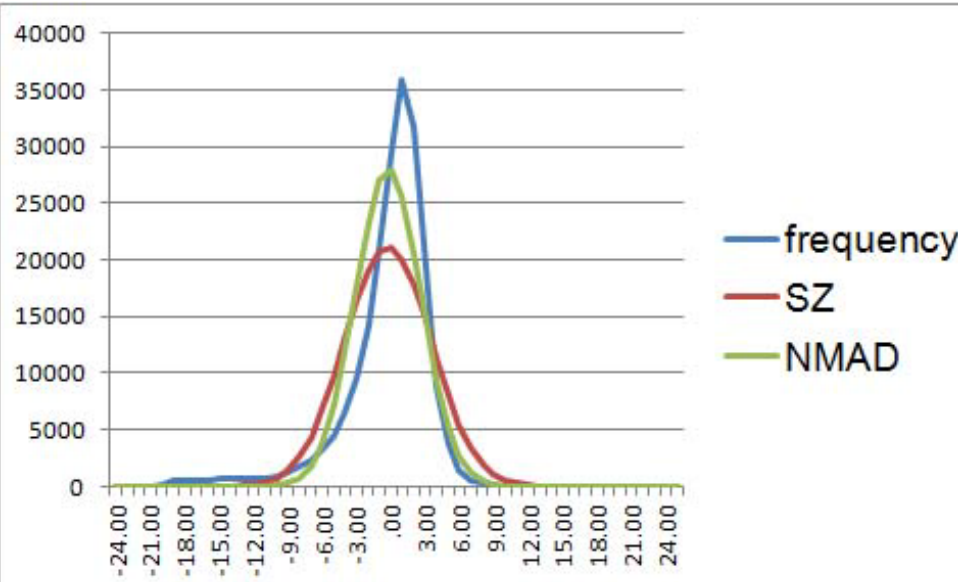

Fig. 4: Frequency distribution with overlaid normal distribution based on SZ and on NMAD

\begin{tabular}{|l|c|c|}
\hline & SZ & NMAD \\
\hline Negative part & $5.13 \mathrm{~m}$ & $5.20 \mathrm{~m}$ \\
\hline Positive part & $2.07 \mathrm{~m}$ & $2.43 \mathrm{~m}$ \\
\hline
\end{tabular}

The CoSSC DSM has been compared with a reference DTM. Of course in such a case the accuracy figures are strongly influenced by buildings and vegetation as it can be seen in the color coded differences (Fig.3) by the yellow up to red color. Also the frequency distribution (Fig. 4) shows this effect by quite higher number of negative differences as corresponding to the normal distribution. If only the positive part of the frequency distribution is used, by theory this just should be dominated by the uncertainty of the TDX height determination because such object points cannot be located below the reference DTM, corresponding the standard deviation based on the positive part of the frequency distribution is just $2.07 \mathrm{~m}$ and the NMAD $2.43 \mathrm{~m}$. In this case the NMAD is larger as the standard deviation. That means the number of larger discrepancies is smaller as corresponding to the normal distribution.

A follow-on investigation focused at open areas that cover about one quarter of the area. For the sake of saving space we do not show any figures here but just give the numbers, which are significantly improved:

\begin{tabular}{|l|c|c|c|c|c|}
\hline RMSZ & bias & SZ & NMAD & LE90 & LE95 \\
\hline $2.67 \mathrm{~m}$ & $0.15 \mathrm{~m}$ & $2.66 \mathrm{~m}$ & $2.57 \mathrm{~m}$ & $4.35 \mathrm{~m}$ & $5.42 \mathrm{~m}$ \\
\hline \multicolumn{5}{|c|}{} \\
\begin{tabular}{|l|c|c|}
\hline \multicolumn{5}{|c|}{ SZ } & NMAD \\
\hline Negative part & $3.49 \mathrm{~m}$ & $4.05 \mathrm{~m}$ \\
\hline Positive part & $1.75 \mathrm{~m}$ & $2.06 \mathrm{~m}$ \\
\hline
\end{tabular}
\end{tabular}

\subsection{Validation versus LIDAR DSM}

For the second test we chose a LIDAR DSM which was available for a part of the city only. In Figures 5 and 6 the Laserscan data and the color coded difference map are shown. Many discrepancies occur at the border of the buildings which are blurred in the radar product. It is worthwhile to consider the area of very poor height values in the lower part (highlighted red in Figure 6), which coincides with lake Maschsee. This region contributes noticeable to the numbers given in the table below. It should be kept in mind that the processing of the future real products of the TanDEM-X mission will take water surfaces into account by masking them out (this was not done in our processing because no water mask is provided for CoSCC data). This means, that such effects are expected not occur anymore.

\begin{tabular}{|c|c|c|c|c|c|}
\hline RMSZ & bias & SZ & NMAD & LE90 & LE95 \\
\hline $5.53 \mathrm{~m}$ & $2.89 \mathrm{~m}$ & $4.72 \mathrm{~m}$ & $3.28 \mathrm{~m}$ & $9.02 \mathrm{~m}$ & $12.22 \mathrm{~m}$ \\
\hline
\end{tabular}

Finally, we discuss qualitatively some effects for the city center area of Hannover. For this purpose we provide two normalized DSM: from the LIDAR data and the CoSSC data (Fig. 7) we subtract the reference DTM. There are several reasons for the blur in case of CoSSC DSM: layover of building superimposes signal from roads and shadow cast behind buildings lead to many noisy areas in cities. It is interesting to look at the difference of both normalized DSM, which is shown in Figure 8. We see clearly the effect of too high elevation for roads in the area in the upper right (red color) close to the main railway station. But there are also many buildings which coincide with too low height (highlighted in blue): we assume that phaseunwrapping problems occurred due to the low height ambiguity of only $16 \mathrm{~m}$.

\section{CONCLUSION AND OUTLOOK}

We have given an overview of the current TanDEM-X mission. This mission will yield a global DEM that will outperform other products like SRTM by far. Since the final DEM is not available yet, we investigated an intermediate product. The outcomes met our expectations. The water mask of the final DEM will be crucial in order to exclude those problematic regions.

\section{ACKNOWLEDGEMENTS}

We thank DLR for providing TerraSAR-X data and Tandem-X data in the framework of a couple of scientific projects. In particular we are grateful for the support of Achim Roth and Birgit Wessel of DLR, who provided not only data but also gave many useful hints that helped a lot. 


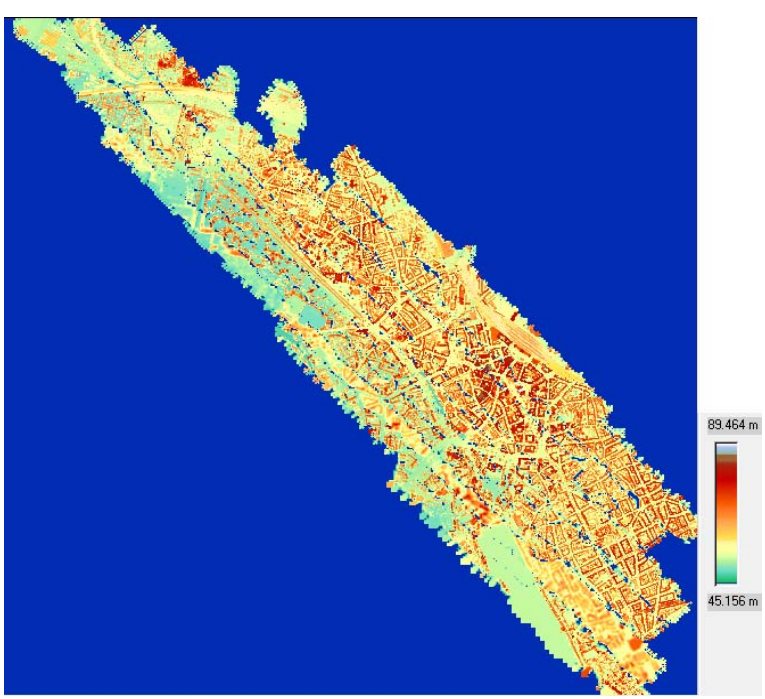

Fig. 5: LIDAR DSM

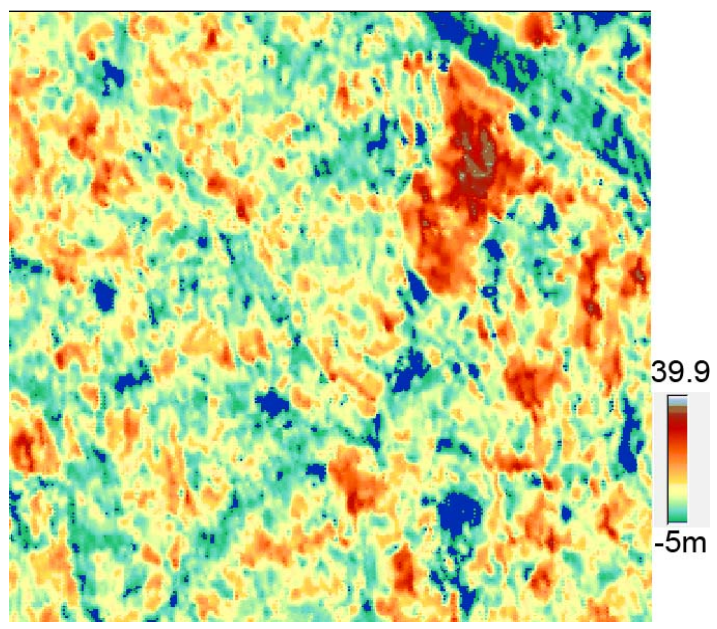

Fig. 7: Difference CoSSC DSM - reference DTM

\section{REFERENCES}

Hanssen R (2001) Radar Interferometry: Data Interpretation and Error Analysis. Kluwer Academic Publishers, Dordrecht, The Netherlands.

Höhle, J. and Höhle, M., 2009. Accuracy assessment of digital elevation models by means of robust statistical methods, ISPRS Journal of Photogrammetry and Remote Sensing, 64, pp. $398-406$

Jacobsen, K., 2008: Satellite image orientation, ISPRS congress Beijing (WG I/5), IntArchPhRS. Vol. XXXVII.

Krieger, G., Moreira, A., Fiedler, H., Hajnsek, I., Werner, M. Younis, M., Zink, M. (2007): TanDEM-X: A satellite formation for high-resolution SAR interferometry. In: IEEE Transactions on Geoscience and Remote Sensing. 45, Nr. 11, 2007, pp. 3317-3341, doi:10.1109/TGRS.2007.900693

Martone, M., Bräutigam, B., Rizzoli, P., Gonzalez, C., Bachmann, M., Krieger, G. (2012): Coherence Evaluation of TanDEM-X Interferometric Data. ISPRS Journal of Photogrammetry and Remote Sensing, vol. 73, pp. 21-29

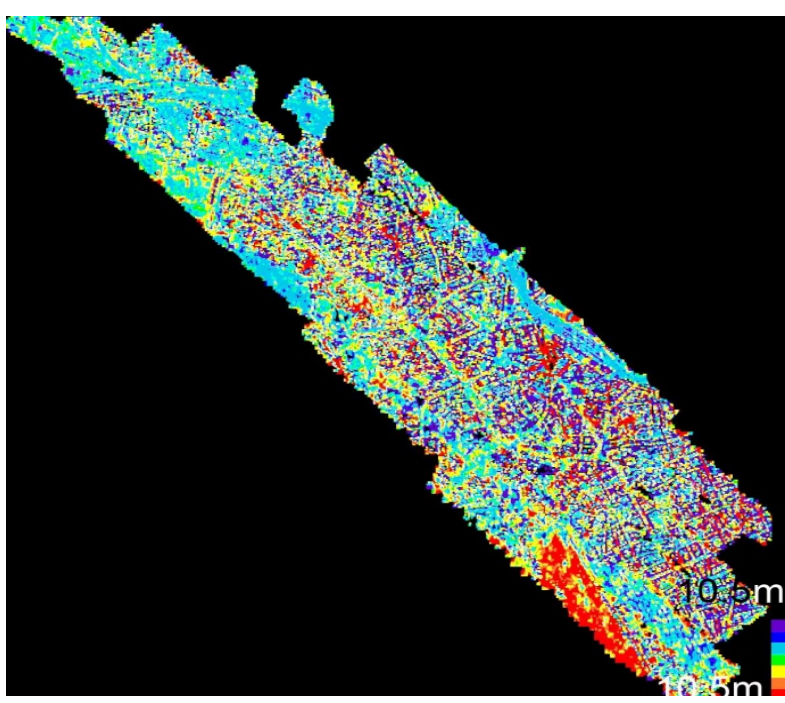

Fig. 6: Difference LIDAR DSM - CoSSC DSM

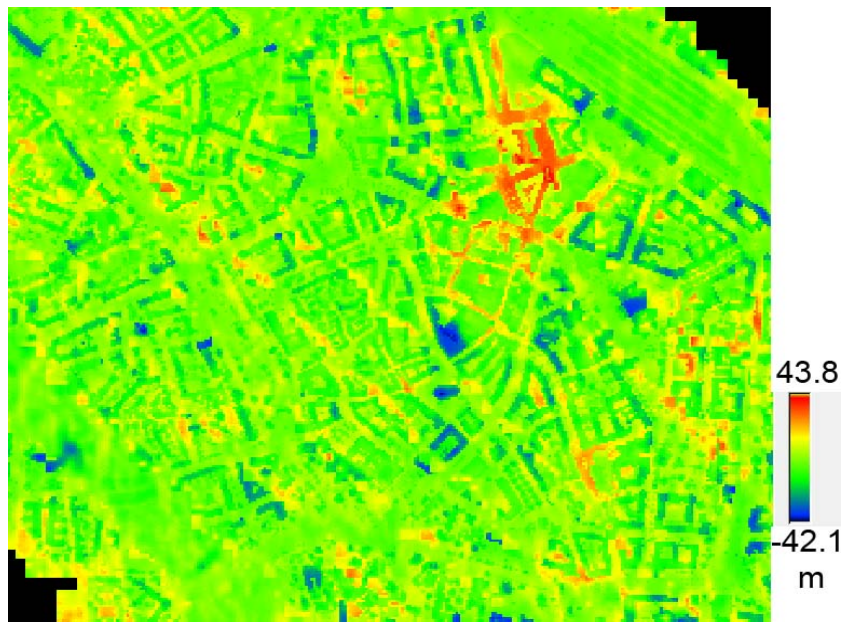

Fig. 8: Difference CoSSC DSM - LIDAR DSM

Rabus, B., Eineder, M., Roth, A., Bamler, R. (2003): The shuttle radar topography mission - a new class of digital elevation models acquired by spaceborne radar, ISPRS Journal of Photogrammetry and Remote Sensing, vol. 57, no. 4 , pp. 241-262.

Soergel, U. (2010): Review of radar remote sensing on urban areas. Radar Remote Sensing of Urban Areas. Springer, Berlin, Germany, pp. 1-47.

TanDEM-X Science Plan (2010, accessed June 2013): https://tandemX-science.dlr.de/pdfs/TD-GS-PL-0069TanDEM-X-Science-Plan_V1_300610.pdf

TanDEM-X DEM Product Specification (2013, accessed June 2013): https://tandemx-science.dlr.de/pdfs/TD-GS-PS0021_DEM-Product-Specification_v2.0.pdf

Wendleder, A., Wessel, B., Roth, A., Breunig, M., Martin, K., Wagenbrenner, S. (2012): TanDEM-X Water Indication Mask: Generation and First Evaluation Results. IEEE Journal of Selected Topics in Applied Earth Observations and Remote Sensing", vol. 6, no. 1, pp. 171-179. 